\title{
El Acceso Abierto como fórmula hacia una Univer- sidad más adaptada al contexto de cultura digital: tendencias y experiencias en el caso español
}

\author{
María SÁNCHEZ GonZÁLEZ \\ Universidad de Málaga \\ m.sanchezgonzalez@uma.es
}

Recibido: 30/07/2012

Aceptado: 23/10/2012

\section{Resumen}

En los últimos años las universidades se han sumado al movimiento Open Access como una de las vías para erigirse en instituciones más abiertas, participativas y adaptadas a las demandas de acceso al conocimiento y a las prácticas y culturas digitales propias de la sociedad-red. El presente artículo recorre sus principales manifestaciones, especialmente en cuanto a iniciativas para la publicación de recursos educativos abiertos y para su puesta a disposición a través de repositorios en red, en el caso español.

Palabras clave: Open Access, Universidad 2.0, Creative Commons, Recursos Educativos Abiertos, Repositorios Institucionales

Open Access as a way towards a University more adapted to the digital culture context: trends and experiences in the Spanish case

\begin{abstract}
In the last years the universities have joined the Open Access movement, as one way to became in institutions more open, participative and adapted to the demands of access to Knowledge and the digital practices and cultures characteristic of the network society. This article presents an overview of the main expressions in the Spanish University, especially regarding initiatives on open educational resources publication and on institutional repositories and other websites for their cataloging.

Keywords: Open Access, University 2.0, Creative Commons, Open Educational Resources, Institutional Repositories

\section{Referencia normalizada}

SÁNCHEZ GONZÁLEZ, María (2012): "El Acceso Abierto como fórmula hacia una Universidad más adaptada al contexto de cultura digital: tendencias y experiencias en el caso español". Estudios sobre el mensaje periodístico. Vol. 18, núm. especial noviembre, págs.: 859-868. Madrid, Servicio de Publicaciones de la Universidad Complutense.
\end{abstract}

Sumario: 1. Introducción. 2. Metodología. 3. Desarrollo; 3.1. Proyectos para la generación de opencourses; 3.2. Píldoras de aprendizaje multimedia en redes interuniversitarias; 3.3. Podcasting y Videocasting; 3.4. Programas para la creación de contenidos digitales; 3.5. Repositorios institucionales y bibliotecas 2.0. 4. Conclusiones; 4.1. Motivaciones para Universidades y docentes; 4.2. Retos para una mayor calidad y aprovechamiento de los REA. 5. Referencias bibliográficas.

\section{Introducción}

Declaraciones internacionales como las de Budapest, Bethesda y Berlín suponen, a comienzos del presente siglo, los primeros pasos del reconocimiento gubernamental del valor de Internet como vía para el acceso abierto, sin restricciones económicas, legales o técnicas, a documentos elaborados por la comunidad científica (Noguerol et al, 2010: web). Se trata, con el consecuente desarrollo de políticas Open Access y mediante alternativas al tradicional sistema de propiedad intelectual como el Copyleft y, 
específicamente, licencias del tipo Creative Commons, de que cada autor esté en disposición de decidir cómo otros pueden consumir y reutilizar sus contenidos en red (Lessig, 2004: 279).

Fenómenos tecnológicos como el software libre y la denominada web 2.0 han impulsado el Acceso Abierto por varias razones. Primero, porque su filosofía ha alcanzado a todos los ámbitos, dando lugar a una nueva cultura digital en la que a tradicionales creadores de contenido, como científicos y académicos, se suman los ciudadanos, capaces de remezclar y reinterpretar, en este contexto, los contenidos de otros (Deuze, 2006). Y segundo porque, aunque la generación de recursos o la implementación de archivos abiertos no requieren necesariamente de estas tecnologías y herramientas, su eclosión disminuye la necesidad de inversión para instituciones que, como las universidades, se han sumado a dicho movimiento.

Así, desde distintos países se vienen poniendo en marcha iniciativas que, haciendo uso casi siempre de las mismas, persiguen un objetivo común: promover el conocimiento haciendo visibles a través de Internet recursos procedentes de la docencia e investigación. Con el tiempo se han adoptado políticas de acceso abierto por defecto, exigiendo a los miembros de la comunidad universitaria la remisión de documentos académicos o científicos para su publicación en repositorios digitales conforme a licencias acordes ${ }^{1}$, e incluso se han desarrollado proyectos orientados a la producción específica de open contents.

Con ello se pretende dar respuesta a las demandas de aprendizaje continuo de unos ciudadanos inmersos en las prácticas de participación, remediación y bricolaje (Deuze, 2006) propias de esta cultura digital. Y para garantizar, más allá, su distribución conforme a una serie de premisas técnicas, jurídicas y didáctico-pedagógicas, estas iniciativas han ido acompañadas de la puesta en marcha de infraestructuras y de recursos de apoyo y asesoramiento docente. En el sentido de que, como apunta Duart (2007: web) "abrir los contenidos de una institución educativa es abrir las puertas de la institución al mundo", y por tanto una "decisión estratégica que conlleva importantes cambios en la dinámica institucional no sólo desde el punto de vista tecnológico, sino también educativo, así como económico y legal".

\section{Metodología}

El objetivo del presente estudio, de carácter exploratorio y cualitativo, es realizar un recorrido, a modo de estado de la cuestión, sobre las manifestaciones Open Access en el contexto universitario español actual.

Dejando a un lado experiencias recientes como los Massive Open Online Courses (MOOCs) iniciados por Siemens y Downes en 2008 desde la Universidad de Manitoba ${ }^{2}$, que comienzan a generalizarse en el ámbito estadounidense a través de plata-

${ }^{1}$ Como ejemplo de mandato Open Access, el adoptado por el MIT en 2009. Cfr. http://infolibraries.mit.edu/scholarly/about/

${ }^{2}$ Cfr. http://pragmaticful.blogspot.com.es/2011/03/que-es-un-mooc.html 
formas como Coursera.org y que aún no han tenido prácticamente reflejo en la universidad española ${ }^{3}$, se consideran dos niveles.

En cuanto, primero, a los Open Contents, se presta especial atención, por su diversidad, a los orientados a la enseñanza-aprendizaje, a los que la UNESCO se refirió por primera vez en 2002 como recursos educativos abiertos o REA (Ehlers y Koskinen, 2011:web) ${ }^{4}$. Atendiendo a su amplitud, van desde open courses o asignaturas completas, compuestos de recursos de distinta naturaleza; hasta fragmentos más pequeños, a modo de píldoras formativas u objetos de aprendizaje en formato textual o multimedia.

En segundo lugar, como Open Archives, además de los repositorios institucionales, como conjunto clasificado de REA y de recursos procedentes de la investigación, conforme a una serie de requisitos que facilitan su recuperación e interoperabilidad en red (Red Alfa, 2007: 15-ss; Santos Hermosa et al, 2012: 137-ss), se consideran aquellos portales online que albergan, como se verá, proyectos específicos de generación de open courses así como los canales en red para la distribución de vídeos o podcast. Mediante metadatos o descriptores, los recursos de estos sitios pueden mostrarse desde los propios repositorios institucionales que actúan entonces como espacios centralizados de búsqueda y enlace a estos repositorios distribuidos.

Partiendo de la anterior diferenciación, y a través del análisis documental y de la observación de los propios sitios online de las universidades españolas, se han detectado y estudiado las tendencias más significativas en ambos niveles para realizar, en fin, una taxonomía de fórmulas, recogiendo y analizando, en cada caso, las experiencias más relevantes puestas en marcha hasta 2012.

\section{Desarrollo}

\subsection{Proyectos para la generación de open courses}

En 2002 el MIT ponía en marcha su OpenCourseWare (http://ocw.mit.edu), un portal web con materiales de sus cursos, organizados por categorías y asignaturas, en abierto y bajo Licencia Creative Commons, con la idea de que fueran usados o reutilizados por educadores y estudiantes. A esta iniciativa pionera se sumarían, a través del consorcio mundial creado en 2006 y de consorcios regionales, universidades de todo el mundo que, desde entonces, han venido desarrollando sus propios OCW.

Es el caso del OCW-Universia (http://ocw.universia.net.es), que agrupaba, a mediados de 2011, a 110 universidades españolas, portuguesas e iberoamericanas cuya producción total superaba 1500 asignaturas (Aranzadi y Capdevila, 2011:130-ss), accesibles, mediante sindicación, desde su propio portal. Cada universidad gestiona su proyecto normalmente desde una Oficina $O C W$, y como incentivo para la publicación de conte-

3 Según datos de la propia plataforma, en octubre de 2012 hay cerca de doscientos de 33 universidades estadounidenses (el doble que un mes atrás) y cerca de 1.700 .000 estudiantes o "courserians". En España, entre los escasos pilotajes cabe citar Unimooc (http://www.iei.ua.es/mooc-emprendimiento/), puesto en marcha en 2012.

${ }^{4}$ En 2011 la UNESCO ha editado, además, con el Commonwealth of Learning, A Basic Guide to Open Educational Resources (OER). Cfr. http://www.col.org/about/whatis/Pages/chronology.aspx 
nidos por el profesorado muchas lanzan convocatorias periódicas que incluyen la concesión de ayudas económicas y su reconocimiento como proyectos de innovación y/o como material publicado, a lo que se suman los Premios Anuales MEC-Universia.

Entre las españolas más veteranas y activas en cuanto a publicación de asignaturas, destacan la Politécnica de Madrid (http://ocw.upm.es/), impulsora de documentación técnica y jurídica en el Consorcio; Alicante (http://ocw.ua.es/), con más de doscientas asignaturas y premiada en los Awards for OCW Excellence de 2011; o Cantabria (http://ocw.unican.es), también galardonada en varias ocasiones, que incluye grados completos, y que lideraba, ese año, el Consorcio en cuanto a número de visitas.

En el caso andaluz, la apuesta por el OCW es desigual en las grandes universidades. Así, mientras Sevilla, aglutinaba, hacia abril de 2012, cerca de 80 asignaturas, en Granada la producción es menor (con unas 15 entonces, la mayoría publicadas entre 2008 y 2009), y en otras como Málaga, con su primera convocatoria de ayudas en 2011, el proyecto está aún en fase emergente. Al otro lado, es relevante el caso de la Universidad Internacional de Andalucía (UNIA), institución de tamaño reducido que viene realizando, desde 2008, convocatorias para la publicación de contenidos procedentes de posgrados y formación permanente, al tiempo que viene incorporando a su portal OCW (http://ocw.unia.es), otros sobre formación al profesorado y creación de contenidos digitales. Para finales de 2012 se espera alcanzar el medio centenar de asignaturas compuestas, además de por recursos de aprendizaje, por otros de ayuda y guía que facilitan su (re)utilización (Sánchez, 2012: web).

\subsection{Píldoras de aprendizaje multimedia en redes interuniversitarias}

También en el marco de proyectos interuniversitarios se vienen publicando en abierto recursos educativos de carácter breve y de naturaleza multimedia, fundamentalmente audios, vídeos, videotutoriales, presentaciones o unidades de aprendizaje interactivas.

Es el caso del Campus Andaluz Virtual, en el que participan las diez universidades públicas andaluzas, con "cápsulas de aprendizaje" como "acciones formativas muy breves, diseñadas para uso individual de forma virtual, sin apoyo de un tutor, que utilizan diferentes técnicas y formatos para la presentación de los contenidos", según aparece en su portal (http://campusvirtualandaluz.es). A mediados de 2012 se contabilizaron unas 50, disponibles también para los estudiantes desde los sistemas de docencia virtual de sus universidades. Desde el propio Consorcio OCW-Universia se propuso la creación de una WebTv Educativa Federada para la difusión de vídeos educativos de escasa duración y su reutilización en distintas plataformas federadas (Aranzadi y Capdevila, 2011: 132), aunque ello aún no ha tenido resultados.

\subsection{Podcasting y Videocasting: Itunes U, Youtube Edu y plataformas de Web-tv} La mayoría de televisiones universitarias españolas utiliza plataformas de la web social, como Vimeo, BlipTV o Youtube para aumentar la visibilidad de sus contenidos, e incluso otras herramientas, como blogs, para su catalogación personalizada ${ }^{5}$. Espe-

5 Es el caso de UNIA-TV (http://blogs.unia.es/uniatv/) de la UNIA, blog donde se muestran organizados los vídeos procedentes de su archivo sobre Blip.tv (http://blip.tv/universidadinternacional-de-andalucia). 
cialmente significativo fue el surgimiento, en 2008, de Youtube EDU (www.youtube.com/edu), al que se sumaron inmediatamente universidades como la Politécnica de Madrid (primera en cuanto a visitas entre 2008 y 2011), la Oberta de Cataluña, la Autónoma de Madrid o IE University. Sus responsables la señalan como magnífica herramienta de proyección social, dada su popularidad, que además permite a los estudiantes interaccionar más allá del aula .

También la disponibilidad en España, desde 2009, de iTunes University (iTunes $U$ ), que posibilita la descarga y sindicación de podcasts académicos universitarios, ha llevado a muchas a crear sus propios canales. La primera, ese mismo año, sería Vigo, con contenidos en sintonía con Uvigo- $T V$, en funcionamiento desde 2004, que incluían 2000 horas de conferencias, clases, debates, entrevistas o eventos en varios idiomas $^{7}$. Y tras el lanzamiento, a inicios de 2012, de una aplicación que permite a los profesores crear cursos completos e interactuar con los inscritos vía streaming, eran ya 11 universidades españolas en Itunes $U$ (Caldevilla y Piñeiro-Otero, 2012:87-88).

Sus contenidos obedecen a dos tipologías de podcasting: educativo, orientado a potenciar el aprendizaje y promovido fundamentalmente por docentes; e institucional, sobre actos o presentaciones, a cargo de gabinetes de comunicación y próximos a las "Relaciones Públicas 2.0.". En medio se situarían contenidos sobre congresos y conferencias (Caldevilla y Piñeiro-Otero, 2012: 90). Desde una perspectiva cuantitativa, los institucionales parecen predominar sobre los educativos, que sin embargo son los más descargados, hecho que tal vez haga a las universidades replantear su estrategia para dar valor añadido a estos canales frente a las televisiones a través de Internet tipo $W e b-t v$, más centradas en contenidos institucionales, congresos y conferencias.

Tendencias similares en cuanto a la combinación de contenidos educativos multimedia con otros sobre eventos académicos, son las denominadas mediatecas, espacios asociados bien a los propios repositorios institucionales, bien a las web de proyectos concretos, como sucede en Prácticas y Culturas Digitales de la UNIA (http://practicasdigitales.unia.es/noticias/mediateca.html). La diferencia es que, además de contenidos propios, agregan otros tomados de la web social cuya licencia lo permite.

\subsection{Programas para la producción de contenidos digitales}

Esta producción propia de contenidos educativos se ha visto favorecida por el apoyo institucional a través de programas nacionales como Profesionales Digitales (http://www.profesionalesdigitales.es), en el que participan, actualmente, 21 univer-

${ }^{6}$ Así, la Universidad Internacional Menéndez Pelayo muestra en YouTube los vídeos resumen de sus actividades en Santander para mantener el debate, online, tras éstas (Cfr. http://www.aprendemas.com/Reportajes/P1.asp?Reportaje=1908). También disponen de canales en Youtube las conferencias TED y sus correspondientes Tedx (http://www.ted.com /tedx); o Ideas que Mueven Andalucía (IMA), web que recopila grabaciones de eventos andaluces destacados, incluyendo los universitarios (http://www.youtube.com/user/imatvconferencias).

${ }^{7}$ Cfr. http://maclovers.universiablogs.net/2009/07/16/universidad-de-vigo-la-primera-hispanaen-itunes-u/ 
sidades públicas cuyos proyectos de Centros de Producción y Experimentación en Contenidos Digitales fueron aprobados. Además de recursos para la creación de aulas virtuales mediante videoconferencia, la mayoría dispone de salas, equipos y software de grabación y postproducción que posibilitan su generación.

Es el caso de la fragUA de la Universidad de Alicante, donde desde julio de 2010 a junio de 2012 se han producido 775 pUA, píldoras formativas de unos 10 minutos en las que se superponen y sincronizan dos señales de vídeo, la de una presentación y la de la imagen del profesor mientras explica sus contenidos. De la parte técnica se encarga el servicio de informática junto con biblioteca, que asesora al profesorado en cuestiones legales. Otro ejemplo pionero es la Universidad de Vigo con sus "píldoras polimedia", de formato similar, centradas en un único concepto clave y que pueden agruparse para crear distintos itinerarios de aprendizaje. En ambas se han desarrollado además herramientas para publicar y distribuir estos audiovisuales desde el campus virtual -el caso de Vértice, en Alicante- y desde plataformas como Itunes y Web-tv- como Pumukit, en Vigo-.

\subsection{Repositorios institucionales y bibliotecas 2.0}

Tras la puesta en marcha de repositorios españoles interuniversitarios, como TDX/TDR (http://www.tdx.cat/) para tesis doctorales en red, el surgimiento de plataformas y recursos de código abierto para la creación de archivos digitales, a modo de Bibliotecas 2.0, como DSpace, GNU Eprints o Fedora, impulsa la creación, en el último lustro, de repositorios propios, de forma que hacia 2011 la mitad de las universidades españolas disponía de uno (Abadal et al, 2011, cit por Santos Hermosa et al, 2012: 140141).

La mayoría de documentos agregados son de investigación (Melero et al, 2009: web), pero la tendencia es ir creando repositorios que incluyan también los contenidos educativos generados por cada universidad. Como ejemplos construidos sobre Dspace el de la UNIA (http://dspace.unia.es/), gestionado por Biblioteca, y que además de tesis, proyectos de investigación y diversas publicaciones académicas da acceso a contenidos del $O C W-U N I A$ y vídeos generados desde $U N I A-T V$. O el de Alicante, creado en 2007 y que en enero de 2012 aparecía en el puesto 51 del ranking mundial de repositorios del CSIC y en el 24 de los Top 100 europeos, por detrás, en el caso universitario español, de la Autónoma de Barcelona y la Politècnica de Catalunya ${ }^{8}$.

Este último, a través de su UPCommons: acceso abierto al conocimiento de la $U P C$, arrancado en 2008, es más bien un portal que enlaza con los diferentes repositorios institucionales de la universidad y que incorpora, además, como complemento y soporte, dos servicios denominados Factoría de Recursos Docentes y Servicio de Propiedad Intelectual (SEPI). No es un caso aislado sino que muchas universidades han creado recientemente portales dinámicos, a modo de mashup, que muestran, entre otros, los recursos más destacados o las últimas incorporaciones a los repositorios ins-

${ }^{8} \mathrm{Cfr}$. http://blogs.ua.es/si/2012/02/15/rua-asciende-hasta-el-puesto-51-del-ranking-web-derepositorios-del-mundo/ 
titucionales. Así, REUNIDO de Oviedo (http://www.unioviedo.es/reunido/) y UCrea de Cantabria (http://repositorio.unican.es/xmlui/), ambas lanzadas en 2012, permiten acceder a su producción científica, hasta el momento dispersa. Otras han incorporado además, como vía de promoción, apartados a sus web institucionales -sirvan como ejemplos el de "Uned en Abierto" o el de "Conocimiento Abierto" en la UOC-, donde recopilan la información clave y dan acceso a los sitios de sus proyectos.

\section{Conclusiones}

\subsection{Motivaciones para Universidades y docentes}

Lejos de una exposición pública desinteresada, como sostienen Pernías y Marco (2007: 51), la publicación de contenidos en abierto produce, tanto a sus autores como a las universidades que los albergan, "innegables beneficios en términos de prestigio académico, social y científico". Los resultados del OCW-MIT, casi una década después de su inicio, evidenciaban, en este sentido, que abrir los contenidos, en lugar de suponer un descenso de matriculaciones, "ha generado un alto retorno en reputación y autoridad para la institución" y "ha atraído nuevos usuarios, aprendices amateurs, previamente no concebidos como público objetivo" (Lara, 2010: web).

Los proyectos REA se conforman por tanto como una interesante vía para "ampliar la participación en la educación superior" así como para "promover el aprendizaje $d u$ rante toda la vida" (OECD, 2010: 14), lo que contribuye al cumplimiento de la responsabilidad social de la Universidad. Y también, en cuanto a la "necesidad de revisar sus procesos, metodologías y contenidos en busca de la mejora de la calidad de la enseñanza impartida" que se genera conforme esta madura (Pernías y Marco, 2007), la publicación en abierto contribuye a la excelencia. Ello porque los autores de contenidos, conscientes de que éstos formarán parte de un escaparate global y de la incidencia de ello en términos de reputación personal, se esmeran para ofrecer su mejor versión (Frías Navarro et al, 2010), y también porque dicha participación supone un cambio de mentalidad que afecta positivamente a su propia docencia.

\subsection{Retos para una mayor calidad y aprovechamiento de los REA}

Sin embargo, al margen de posibles mejoras en su gestión técnica y jurídica, la falta de estrategias políticas y organizativas acordes continúa suponiendo una barrera para el desarrollo de proyectos de Open Content realmente utilizados por docentes y estudiantes e integrados en las prácticas educativas universitarias. Ante la reseñada mezcla de contenidos educativos con institucionales en buena parte de los archivos, y donde cabría preguntarse si son meros elementos de marketing (Freire y Shuch, 2010: 91), cabe fomentar, para ello, alternativas como los denominados Repositorios Institucionales de Contenido Educativo (RICE). Esto es, repositorios específicamente orientados a la docencia, abiertos a la comunidad universitaria y conectados con las plataformas de gestión del aprendizaje y el resto de sistemas de la institución, de forma que además de su reutilización se facilite "la reelaboración o elaboración colaborativa de los materiales, configurando un entorno colaborativo de trabajo entre los profesores en el que los alumnos cuenten con más y mejores medios para apoyar su aprendizaje" (Bueno de la Fuente y Hernández Pérez, 2011: web). 
Entretanto, proyectos clásicos, como los del MIT, con el lanzamiento, a inicios de 2012, de un prototipo de open course que permite obtener certificación gratuita a quien avale, tras seguirlo, ciertos conocimientos (http://mitx.mit.edu), parecen estar, como el propio ecosistema digital en el que se sitúan, en beta permanente. Al tiempo que nuevas iniciativas, como los referidos $M O O C$, que combinan las posibilidades de la web 2.0 y de los open contents para un aprendizaje permanente social y colaborativo, muestran que otros modelos educativos son posibles. Tal vez la oportunidad de la Universidad -y la clave para su función social en este contexto de revisión de sistemas de reputación y acceso al conocimiento-, no esté ya tanto en la mera creación de contenidos en abierto sino en ejercer, siguiendo la terminología de Freire (2008: 55), una especie de Comisariado digital, como espacio abierto y nodo de referencia, o como "mediadora para ofrecer los contextos de participación, interacción e innovación que necesita el bricolaje del conocimiento colaborativo en la cultura digital" (Lara, 2009: 20).

\section{Referencias bibliográficas}

ARANZADI, Pedro y CAPDEVILA, Ramón (2011): “Open Course Ware, recursos compartidos y conocimiento distribuido". La Cuestión Universitaria, $\mathrm{n}^{\circ} 7$, pp. 126133: http://www.lacuestionuniversitaria.upm.es/web/grafica/articulos/imgs_boletin _7/pdfs/LCU-7-13.pdf. [Fecha de consulta: 5 de agosto de 2012]

BUENO DE LA FUENTE, Gema y HERNÁNDEZ PÉREZ, Toni (2011): “Estrategias para el éxito de los repositorios institucionales de contenido educativo en las bibliotecas digitales universitarias". Revista Textos universitaris de biblioteconomia i documentaci, $\mathrm{n}^{\mathrm{o}} 26$ : http://www.ub.edu/bid/26/bueno2.htm. [Fecha de consulta: 5 de agosto de 2012]

CALDEVILLA, David y PIÑEIRO-OTERO, Teresa (2012): "Relaciones Públicas 2.0. iTunes University como plataforma para la interacción de las Universidades españolas con sus públicos", en AIRP (Ed.): Actas del VII Congreso Internacional de Investigación en Comunicaciones Públicas, Relaciones públicas. El diálogo de las organizaciones. Sevilla, 21-23 marzo 2012, pp.79-96: http://www.airrpp.org /Actas_VII_Congreso_AIRP_Sevilla2012.pdf [Fecha de consulta: 10 de octubre de 2012]

CENTRO PARA LA INVESTIGACIÓN E INNOVACIÓN EDUCATIVAS, OECD (coord.) (2010): El conocimiento libre y los recursos educativos abiertos. Junta de Extremadura: http://www.oecd.org/dataoecd/44/10/42281358.pdf. [Fecha de consulta: 26 de julio de 2012]

DEUZE, Mark (2006): "Participation, Remediation, Bricolage. Considering Principal Components of a Digital Culture". The Information Society. $\mathrm{n}^{\mathrm{o}} 22$, pp. 63-75.

DUART, José María (2007): “Educación abierta en la red”. Revista de Universidad y Sociedad del Conocimiento, vol. 4, $\mathrm{n}^{\circ} 1 \mathrm{http} / / / \mathrm{www} . u o c . e d u /$ rusc/4/1/esp/editorial.html. [Fecha de consulta: 2 de junio de 2012] 
EHLERS, Ulf-Daniel y KOSKINEN, Tapio (2011): "De los recursos a las prácticas. Editorial de Educación abierta: Transformar las prácticas educativas". Elearning Papers, $\mathrm{n}^{\mathrm{o}}$ 23: http://www.elearningeuropa.info/es/paper/elearning-papers-n-23educacion-abierta-transformar-las-practicas-educativas. [Fecha de consulta: 7 de agosto de 2012]

FREIRE, Juan (2008): “Conocimiento y usuarios en la cultura Digital". Revista FRC, $\mathrm{n}^{\mathrm{o}} \quad 16, \quad$ pp. 53-57: http://www.fcampalans.cat/uploads/publicacions/pdf /13.jfreire.pdf [Fecha de consulta: 4 de mayo de 2012]

FREIRE, Juan y SCHUCH BRUNET, Karla (2010): "Políticas y prácticas para la construcción de una universidad digital". La Cuestión Universitaria, $\mathrm{n}^{\mathrm{o}} 6$, pp. 85$94 \mathrm{http}: / /$ www.lacuestionuniversitaria.upm.es/web/grafica/articulos/imgs_boletin_6/pdfs /LCU-6-7.pdf. [Fecha de consulta: 8 de agosto de 2012]

FRÍAS NAVARRO, María Dolores, PASCUAL LLOBELL, Juan, MONTERDE i BORT, Héctor y PASCUAL SOLER, Marcos (2010): Impacto del Open Course Ware $(O C W)$ en los docentes universitarios. Programa de "Estudios y Análisis" (EA2009-0168), Ministerio de Educación: http://www.uv.es/impacocw/impactoOCWValencia.pdf. [Fecha de consulta: 20 de julio de 2012]

LARA, Tíscar (2009): "El papel de la Universidad en la construcción de su identidad digital, en Cultura digital y prácticas creativas en educación [monográfico en línea]". Revista de Universidad y Sociedad del Conocimiento (RUSC), vol.6, $\mathrm{n}^{\circ} 1$ : http://rusc.uoc.edu/ojs/index.php/rusc/article/view/25/19. [Fecha de consulta: 23 de julio de 2012]

LARA, Tíscar (2010): “Contenidos abiertos en Educación Superior: ¿modelo sostenible?" Tiscar. com, 20 de mayo de 2010: http://tiscar.com/2010/05/20/contenidosabiertos-en-educacion-superior-\%C2\%BFmodelo-sostenible/. [Fecha de consulta: 6 de agosto de 2012]

LESSIG, Lawrence (2004): Free culture. How big media uses technology and the law to lock down culture and control creativity. New York, Penguin Group. http://www.free-culture.cc/freeculture.pdf. [Fecha de consulta: 26 de julio de 2012]

MELERO, Remedios et al (2009): "The situation of open access institutional repositories in Spain: 2009 report". Information research, vol. 14, $\mathrm{n}^{\circ}$ 4: http://InformationR.net/ir/14-4/paper415.html. [Fecha de consulta: 24 de mayo de 2012.

NOGUEROL, Ana, HERRANZ, Rafael y ROSÓN, Tomás (2010): “Introducción al Open Access. Materiales del Máster en Economía Digital de la Escuela de Organización Industrial". Savia, EOI. http://www.eoi.es/savia/pubman/item/eoi:52473:3 /component/eoi:52471/EOI_OpenAccess_2010.pdf. [Fecha de consulta: 6 de agosto de 2012]

PERNÍAS, Pedro y MARCO, Manuel (2007): "Motivación y valor del proyecto OpenCourseWare: la universidad del siglo XXI, en Contenidos educativos en abierto" [monográfico en línea]. Revista de Universidad y Sociedad del Conocimiento (RUSC), vol. 4, $\mathrm{n}^{\circ} 1$ : http://www.uoc.edu/rusc/4/1/dt/esp/pernias_marco.pdf. [Fecha de consulta: 6 de agosto de 2012] 
RED ALFA (2007): Directrices para la creación de repositorios institucionales en universidades y organizaciones de educación superior. Pontificia Universidad Católica de Valparaíso. Santiago:http://www.sisbi.uba.ar/institucional/proyectos /internacionales/Directrices_RI_Espa_ol.pdf. [Fecha de consulta: 6 de agosto de 2012]

SÁNCHEZ GONZÁLEZ, María (2012). "Iniciativas para el conocimiento abierto en las Universidades. El caso del OpenCourseWare-UNIA". Congreso Virtual Mundial de e-Learning en español. Data Media et al /Cátedra UNESCO de Educación a Distancia UNED. En http://www.congresoelearning.org, del 1 al 12 de octubre de 2012.

SANTOS-HERMOSA, Gema, FERRAN-FERRER, Núria y ABADAL, Ernest (2012): "Recursos educativos abiertos: repositorios y uso". El profesional de la información, v. 21, $\mathrm{n}^{\mathrm{o}}$ 2, pp. 136-145: http://dx.doi.org/10.3145/epi.2012.mar.03. [Fecha de consulta: 6 de agosto de 2012]

\section{María SÁNCHEZ GONZÁLEZ}

Universidad de Málaga/ Universidad Internacional de Andalucía

m.sanchezgonzalez@uma.es/m.sanchez@unia.es

Datos académicos y profesionales

Profesora asociada doctora en Periodismo e investigadora en la Universidad de Málaga, actividad que compagina con su trabajo como coordinadora docente del Área de Innovación en la Sede Tecnológica de la Universidad Internacional de Andalucía

Dirección postal

Universidad Internacional de Andalucía

c/ Severo Ochoa, 16-20 Conjunto Málaga-Business Park, Edif. Estepona

Parque Tecnológico de Andalucía 29590 Málaga (España)

Tel. +34 952028413/11 